\title{
Final Report DE-FG02-89ER14017
}

\author{
for the period 5/1/89 to $9 / 30 / 04$
}

"Flux Flow, Pinning, and Resistive Behavior in Superconducting Networks"

\author{
PI: Stephen Teitel \\ Deparment of Physics and Astronomy \\ University of Rochester \\ Rochester, New York 14627
}

No unexpended funds remain. 


\section{RESEARCH UNDER DOE SUPPORT 1989-2004}

The PI has carried out numerical investigations to understand the critical behavior of interacting quantized vortices and vortex lines in superconducting networks, films, and three dimensional bulk samples. An emphasis has been to explain phenomenological behavior in the "high- $T_{c}$ " copper-oxide superconductors and related model systems. During the fifteen

years of DOE support, 35 publications have appeared in refereed journals, and four additional manuscripts have been recently submitted for publication (see section II). These publications have received a total of 788 citations according to the ISI Web of Science Citation Index. This work has resulted in invited presentations at 20 international conferences and workshops (see section III), including 4 invited talks at the March Meeting of the American Physical Society, the largest general international conference of the condensed matter physics community. In 2000 the PI was elected a Fellow of the American Physical Society for his work on topics supported by the DOE. Albert Wang, a Rochester undergraduate who worked with the PI, was named one of three finalists for the 2002 Apker Prize of the American Physical Society, a national prize for undergraduate research. Below are summarized some of the highlights of this work. Citations refer to the list of papers in section II.

\section{A. Vortex Line Fluctuations in Bulk Superconductors}

The PI was the first to introduce the uniformly frustrated three dimensional XY model as a model system for studying vortex line fluctuations in high temperature superconductors (paper 7 1991, 78 citations). This model treats vortex line interactions in a natural way, allowing vortex line cutting, vortex line overhangs, and thermally excited closed vortex line loops with no additional ad hoc assumptions. Our numerical simulations explored phase coherence and vortex lattice melting in the presence of an applied magnetic field for models with isotropic and anisotropic couplings (paper 11, 1993, 92 citations; paper 12, 1994, 67 citations; paper 19, 1996, 6 citations; paper 22, 1997, 26 citations). We have also explored in detail behavior in the vortex line liquid above melting, characterizing the vortex line structure function (paper 9, 1992, 18 citations), demonstrating a simple scaling of the correlation length with magnetic field and system anisotropy (paper 26, 1999, 12 citations), and most recently finding evidence against a theoretically proposed vortex loop "blowout" transition (paper 30, 2003).

We also considered the interacting vortex line model dual to the XY model, to extend our work to the case of a finite magnetic penetration length, carrying out analytical studies (paper 13, 1994, 15 citations) as well as numerical simulations (paper 18, 1995, 37 citations; paper 23, 1997, 19 citations). Our work was the first to define clear measures of superconducting phase coherence by looking at the wavevector dependent helicity modulus.

We have also studied the behavior of bulk superconductors at zero applied magnetic field. We carried out one of the first accurate finite size scaling analyses of the ordinary 3D XY model 
(paper 1, 1989, 58 citations). To incorporate magnetic field fluctuations we later studied the 3D XY model with a fluctuating vector potential and explicitly showed, contrary to some recent theoretical claims, that the coherence length and the magnetic penetration length diverge with the same critical exponent and that this exponent was the same as that of the ordinary 3D XY model (paper 25, 1998, 31 citations). Most recently we have explored the case of the zero field transition in the presence of random columnar defects (paper 34, 2004). Our simulations found the critical behavior to cross over to one with anisotropic scaling, and the equilibrium critical exponents which we found are in reasonable agreement with experiments by Kötzler and co-workers. Our work on this model also has applications to the quantum phase transition in the 2D boson Hubbard model.

Finally, we have carried out simulations of the uniformly frustrated 3D XY model with uncorrelated randomness in the bond strengths, in order to model a bulk high temperature superconductor in the presence of random point pinning (paper 29, 2001, 25 citations). Such disorder is always present in high- $T_{c}$ materials. We found that as the disorder strength increases, the vortex lattice melting transition remains first order and continues down to low temperatures, rather than ending at a critical point as had been suggested by experiments. Subsequent experiments on BSCCO by the group of Zeldov and later others, have provided support for this picture. We have also investigated the point disordered model in the limit of extremely weak interlayer couplings, and find that the "vortex slush" phase found in simulations by Nonomura and $\mathrm{Hu}$ appears to be an artifact of finite size effects rather than a true thermodynamic phase (paper 36, 2004).

\section{B. Point Vortices in Two Dimensions - the Lattice Coulomb Gas}

Investigations of the classical neutral integer lattice Coulomb gas have been carried out as a model for interacting point vortices in two dimensional superconducting networks. The PI was the first to explicitly vary the chemical potential for creating charge-anticharge pairs to study the behavior of the Kosterlitz-Thouless theory at high vortex densities. Instead of finding a predicted first order transition, we found that the high density liquid becomes unstable to an ordered charge-anticharge lattice (paper 2, 1990, 29 citations; paper 6, 1991 18 citations; paper 10, 1992, 42 citations; paper 21, 1997, 3 citations). This work also has been of relevance to models of Coulombic criticality in ionic fluids. We have also studied the Coulomb gas analog of the fully frustrated XY model (paper 16, 1994, 35 citations) and the same model with positional disorder (paper 27, 1999, 4 citations), as well as the driven Coulomb gas to compute the dynamic critical exponent (paper 15, 1994, 18 citations).

Using the classical fractional lattice Coulomb gas as a model, the PI was the first to simulate the melting of a dilute vortex lattice in a superconducting network, and to test key predictions of the Halperin-Nelson theory of $2 \mathrm{D}$ melting on a periodic substrate. We were the first to observe the predicted floating solid at intermediate temperatures between the pinned solid and liquid phases (paper 14, 1994, 37 citations; paper 17, 1995, 38 citations; paper 20, 1996, 2 citations). We also carried out the first detailed numerical investigation 
of the unpinning transition from the pinned to floating solid (paper 28, 2001, 3 citations), finding a first order transition rather than the predicted second order Kosterlitz-Thouless transition.

The formation of a frozen, disordered, glass state in the absence of any intrinsic random impurities is a fundamental unresolved problem in condensed matter physics. It was originally suggested in simulations by Halsey that incommesurability effects can drive the formation of such a glassy state for an incommensurate density of vortices in a periodic network. By simulating a sequence of rational approximates, the PI showed that the glassy state found by Halsey was a direct consequence of the particular dynamics he used; instead of a glass transition, there is rather a true first order equilibrium phase transition to an ordered stripped structure, with anisotropic vortex mobility (paper 24, 1998, 20 citations).

We have studied the fully frustrated $(f=1 / 2)$ 2D XY model consisting of a checkerboard pattern of vortices on a square grid of sites and confirmed a recent prediction by Korshunov for a kink-antikink unbinding transition of corners on the domain walls associated with the broken discrete degeneracy of the ordered state (paper 35, 2005).

Most recently we have investigated the nature of spatial ordering in driven steady states far from equilibrium in the lattice Coulomb gas. We have shown that a commonly used driven diffusive Monte Carlo alogrithm contains unphysical randomness that always disorders a moving state. We have developed a new continuous time Monte Carlo algorithm that correctly reduces to Langevin continuum dynamics in an appropriate limit, and thus gives more physically correct results. We find that the low temperature state of a finite sized system of driven vortices is a smectic state, in which particles flow in periodically spaced channels oriented parallel to the driving force. Motion in different channels is correlated over only a finite length scale $\xi_{\perp}$. Uusing detailed finite size scaling analyses, however, we find that this smectic state is ultimately unstable to an anisotropic liquid on very large length scales. We have also found surprising evidence for a hexatic liquid state for the case of the driven system on a square grid which we explain as a specifically non-equilibrium effect (paper 31, 2004; paper 39, 2005).

\section{Other projects}

Unrelated to the primary thrust in superconductivity, we have also investigated the structural properties of gold nanoclusters. These have attracted considerable attention due to their unique optical and mechanical properties, and have found application in fields such as nano-lithography, catalysis, nano-bioelectronic devices, and ion detection. Using molecular dynamic simulations and a realistic "glue potential" to model the interaction between gold atoms in clusters of a few thousand atoms, we have investigated two related issues. First we

have cooled a liquid cluster to find the preferred equilibrium shape and find that a MacKay icosahedron forms. The lower free energy of the exposed $\{111\}$ facets compensates for the internal grain boundaries needed to form the icosahedral structure. We then study this 
structure upon reheating through melting and find that it retains its icosahedral shape until about $200 \mathrm{~K}$ below melting, when diffusion of atoms along the vertices and edges of the cluster starts to become significant. We compute for the first time the average cluster shape and find that, while the order of the $\{111\}$ facets persists right up to melting, the size of these facets steadily shrink until the cluster is almost spherical in shape just before melting (paper 33, 2004; paper 37, 2004).

The second problem we have considered is the heating of a nanocluster with an initial rod-like shape, as in recent laser heating experiments. We find, in agreement with the experiments, that the nanorod undergoes a shape and structural transformation at a temperature well below the rod melting. We have identified the onset of this transformation as due to the roughening of $\{110\}$ facets on the initial surface of the rod. As the surface instability sets in, the rod relaxes so as to reorganize the surface into more stable $\{111\}$ facets. More surprisingly, we find that the fcc atoms in the interior of the rod completely reorganize themselves to a new orientation to be consistent with the newly formed $\{111\}$ facets (paper $38,2004)$. Both works illustrate the competition between surface and bulk in determining stable nanocluster structures. 


\section{PUBLICATIONS ACKNOWLEDGING DOE SUPPORT (1989-2004)}

preprints may be obtained at http://www.arXiv.org/find/cond-mat/1/au:+Teitel_S/0/1/0/all/0/

1. Ying-Hong Li and S. Teitel

Finite-Size Scaling Study of the Three-Dimensional Classical XY Model

Phys. Rev. B 40, 9122 (1989)

2. Jong-Rim Lee and S. Teitel

New Critical Behavior in the Dense Two-Dimensional Classical Coulomb Gas

Phys. Rev. Lett. 64, 1483 (1990)

3. Ying-Hong Li and S. Teitel

Three-Dimensional Random XY Model: Application to the Superfluid Transition of ${ }^{4}$ He in Porous Media

Phys. Rev. B 41, 11388 (1990)

4. John Chiu and S. Teitel

The Effect of Dynamics on Damage Spreading in the Two-Dimensional Classical XY

Model

J. Phys. A 23, L891 (1990)

5. Ying-Hong Li and S. Teitel

Flux-Flow Resistance in Frustrated Josephson-Junction Arrays

Phys. Rev. Lett. 65, 2595 (1990)

6. Jong-Rim Lee and S. Teitel

Dense Two-Dimensional Classical Coulomb Gas on a Triangular Lattice

Phys. Rev. Lett. 66, 2100 (1991)

7. Ying-Hong Li and S. Teitel

Vortex-Line-Lattice Melting, Vortex-Line Cutting, and Entanglement in Model High$\mathrm{T}_{c}$ Superconductors

Phys. Rev. Lett. 66, 3301 (1991)

8. Ying-Hong Li and S. Teitel

The Effect of Random Pinning Sites on Behavior in Josephson-Junction Arrays

Phys. Rev. Lett. 67, 2894 (1991)

9. Ying-Hong Li and S. Teitel

Structure of a Dense Vortex Line Liquid in a Model High- $\mathrm{T}_{c}$ Superconductor

Phys. Rev. B 45, 5718 (1992)

10. Jong-Rim Lee and S. Teitel

Phase Transitions in Classical Two-Dimensional Lattice Coulomb Gasses

Phys. Rev. B 46, 3247 (1992)

11. Ying-Hong Li and S. Teitel

Vortex-Line Fluctuations in Model High-Temperature Superconductors

Phys. Rev. B 47, 359 (1993)

12. Ying-Hong Li and S. Teitel

Phase Transitions and Vortex-Line Entanglement in a Model High-Temperature Superconductor

Phys. Rev. B 49, 4136 (1994) 
13. Tao Chen and S. Teitel

Helicity Modulus and Meissner Effect in a Fluctuating Type-II Superconductor

Phys. Rev. Lett. 72, 2085 (1994)

14. M. Franz and S. Teitel

Vortex Lattice Melting in 2D Superconductors and Josephson Arrays

Phys. Rev. Lett. 73, 480 (1994)

15. Jong-Rim Lee and S. Teitel

Driven Diffusion in the Two-Dimensional Lattice Coulomb Gas: A Model for Flux Flow in Superconducting Networks

Phys. Rev. B 50, 3149 (1994)

16. Jong-Rim Lee

Phase Transitions in the Two-Dimensional Classical Lattice Coulomb Gas of HalfInteger Charges

Phys. Rev. B 49, 3317 (1994)

17. M. Franz and S. Teitel

Vortex-Lattice Melting in Two-Dimensional Superconducting Networks and Films

Phys. Rev. B 51, 6551 (1995)

18. Tao Chen and S. Teitel

Superconducting Coherence in a Vortex Line Liquid: Simulations With Finite $\lambda$

Phys. Rev. Lett. 74, 2792 (1995)

19. Tao Chen and S. Teitel

Comment on Longitudinal Superconductivity in Vortex-Line Phases: A Monte Carlo Study

Phys. Rev. Lett. 76, 714 (1996)

20. M. Franz and S. Teitel

Vortex Lattice Melting in 2D Superconducting Networks

in Proceedings of the ITCP Workshop on Josephson Junction Arrays Physica B 222, 287 (1996)

21. Pramod Gupta and S. Teitel

Phase Diagram of the Two-Dimensional Lattice Coulomb Gas

Phys. Rev. B 55, 2756 (1997)

22. Tao Chen and S. Teitel

Phase Transitions in High Tc Superconductors and the Anisotropic 3D XY Model

Phys. Rev. B 55, 11766 (1997)

23. Tao Chen and S. Teitel

Helicity Modulus and Fluctuating Type II Superconductors

Phys. Rev. B 55, 15197 (1997)

24. Pramod Gupta, S. Teitel and Michel J. P. Gingras

Glassiness vs. Order in Densely Frustrated Josephson Arrays

Phys. Rev. Lett. 80, 105 (1998)

25. Peter Olsson and S. Teitel

Critical Behavior of the Meissner Transition in the Lattice London Superconductor

Phys. Rev. Lett. 80, 1964 (1998) 
26. Peter Olsson and S. Teitel

Correlation Lengths in the Vortex Line Liquid of a High $T_{c}$ Superconductor

Phys. Rev. Lett. 82, 2183 (1999)

27. Pramod Gupta and S. Teitel

Positional Disorder in the Fully Frustrated Josephson Array: Random Gaussian Phase

Shifts in the Fully Frustrated 2D XY Model

Phys. Rev. Lett. 82, 5313 (1999)

28. Violeta Gotcheva and S. Teitel

Depinning Transition of a Two Dimensional Vortex Lattice in a Commensurate Periodic Potential

Phys. Rev. Lett. 86, 2126 (2001)

29. P. Olsson and S. Teitel

Disorder Driven Melting of the Vortex Line Lattice

Phys. Rev. Lett. 87, 137001 (2001)

30. Peter Olsson and S. Teitel

Search for a Vortex Loop Blowout Transition in a Type-II Superconductor in a Finite Magnetic Field

Phys. Rev. B 67, 144515 (2003)

31. Violeta Gotcheva, Albert T. J. Wang and S. Teitel

Lattice Gas Dynamics; Application to Driven Vortices in Two Dimensional Superconductors

Phys. Rev. Lett. 92, 247005 (2004)

32. T. Goodman and S. Teitel

Roughness of a Tilted Anharmonic String at Depinning

Phys. Rev. E 69, 62105 (2004)

33. Yanting Wang, S. Teitel and Christoph Dellago

Melting and Equilibrium Shape of Icosahedral Gold Nanoparticles

Chem. Phys. Lett. 394, 257 (2004)

34. Anders Vestergren, Mats Wallin, Hans Weber and S. Teitel

Effect of Columnar Disorder on the Superconducting Transition of a Type-II Superconductor in Zero Applied Magnetic Field

Phys. Rev. B 70, 54508 (2004)

35. Peter Olsson and S. Teitel

Kink-Antikink Unbinding Transition in the Two Dimensional Fully Frustrated XY Model

Phys. Rev. B, 71, 104423 (2005)

36. P. Olsson and S. Teitel

Comment on "Effects of Point Defects on the Phase Diagram of Vortex States in High$\mathrm{T}_{c}$ Superconductors in the B || c Axis"

Phys. Rev. Lett., accepted (2004 preprint cond-mat/0404473)

37. Yanting Wang, S. Teitel and Christoph Dellago

Melting of Icosahedral Gold Nanocluster From Molecular Dynamics Simulations

J. Chem. Phys., accepted (2004 preprint cond-mat/0408458)

38. Yanting Wang, S. Teitel and Christoph Dellago

Surface Driven Bulk Reconstruction of Gold Nanorods

Phys. Rev. Lett., submitted (2004 preprint cond-mat/0410454) 
39. Violeta Gotcheva, Yanting Wang, Albert T. J. Wang and S. Teitel

Continuous Time Monte Carlo and Spatial Ordering in Driven Lattice Gases: Application to Driven Vortices in Superconducting Networks

Phys. Rev. B, submitted (2005 preprint cond-mat/0503527)

\section{INVITED CONFERENCE PARTICIPATION FROM DOE SUPPORT}

1. March Meeting of the American Physical Society, Cincinnati, OH, 3/21/91 Theory of Flux Flow Resistance in Josephson Junction Arrays (invited talk)

2. Workshop on: "Inertial Sensors: An Application of the Physics of Supercondutors", Vail, CO, 6/10/92

Vortex Line Fluctuations in High Temperature Superconductors (invited talk)

3. Adriatico Research Conference on: "Vortex Fluctuations in Superconductors", ICTP Trieste, Italy, 8/17/93

Vortex Line Fluctuations and Phase Transitions in High Temperature Superconductors (invited talk)

4. March Meeting of the American Physical Society, Pittsburg, PA, 3/25/94

Phase Transitions and Vortex Line Fluctuations in 3D Superconductors (invited talk)

5. Josephson Junction Arrays Workshop, Jackson Hole, WY, 5/27/95

Phase Transitions in Three Dimensional Josephson Junction Arrays (invited talk)

6. International Workshop on Vortex Dynamics, Lake Forrest, IL, 6/24/95 (participation by invitation only)

Coherence in a Vortex Line Liquid (invited talk)

7. ICTP Workshop on Josephson Junction Arrays, Trieste, Italy, 8/7/95

Vortex Lattice Melting in Two Dimensional Josephson Junction Arrays (invited talk)

8. Workshop on Computational Superconductivity, Argonne, IL, 3/26/96

Vortex Lattice Melting in Two Dimensional Josephson Junction Arrays (invited talk)

9. XIV Sitges Confereence on "Complex Behavior of Glassy Systems", Barcelona, Spain, $6 / 10 / 96$

Equlibrium Phase Transitions in Josephson Junction Arrays (invited talk)

10. NATO/Adriatico Advanced Research Workshop on: "Fluctuation Phenomena in High Critical Temperature Superconducting Ceramics", ICTP Trieste, Italy, 8/10/96

Vortex Line Fluctuations and Phase Transitions in Type II Superconductors (invited talk)

11. 4th International Workshop on Vortex Matter, Monte Verita, Ascona, Switzerland, 6/15/97 (participation by invitation only)

Critical Behavior of the $\mathrm{H}=0$ Meissner Transition (poster)

Effect of Boundary Conditions on the De-coupling Transition (poster)

Chairman, discussion session on simulations

12. International Workshop on Vortex Physics in High Temperature Superconductors, Hachimantai, Japan, 6/21/98 (participation by invitation only)

Correlation Lengths at the Vortex Lattice Melting Transition (poster) 
13. International Workshop on Vortex Physics in High Temperature Superconductors, Stanford, CA, 6/20/99 (participation by invitation only)

Depinning Transition of a Two Dimensional Vortex Lattice in a Commensurate Pinning Potential (poster)

14. March Meeting of the American Physical Society, Minneapolis, MN. 3/23/00

Phase Transitions in High $\mathrm{T}_{c}$ Superconductors and the Anisotropic 3D XY Model (invited talk)

15. The Joint Vortex-Physics and ESF-Vortex Matter Workshop, Lunteren, The Netherlands, 8/27/00 (participation by invitation only)

The Effect of Uncorrelated Random Point Disorder on the Vortex-Line Lattice Melting Transition (poster)

16. Gordon Reasearch Conference on Condensed Matter Physics - Afternoon Workshop on Vortex Matter, New London, CT, 6/18/01

Numerical Simulations of Disorder Driven Melting of the Vortex Line Lattice (invited talk)

17. ESF Workshop on Disordered Systems at Low Temperatures and their Topological Properties, Espoo, Finland, 1/17/02

Disorder Driven Melting of the Vortex Line Lattice (invited talk)

18. March Meeting of the American Physical Society, Indianapolis, IN, 3/21/02

Disorder Driven Melting of the Vortex Line Lattice (invited talk)

Students Lunch with the Experts: discussion leader for lunch table on Vortex Matter (invited)

19. Joint 9th International Workshop on Vortex Dynamics and Vortex Matter (ESF), Oléron, France, 6/22/03 (participation by invitation only)

Search for a Vortex Loop Blowout Transition in a type-II Superconductor in a Finite Magnetic Field (poster)

Vortex glass transition and phase diagram of a frustrated 3D XY model with disorder (poster)

20. Tenth International Vortex State Studies Workshop, Mumbai, India, 1/9/05 (participation by invitation only)

Phase Diagram of Model Point Disordered Superconductor (invited talk) Columnar Disorder and the Zero Field Superconducting Transition (poster)

Steady States of Driven Vortices in a Periodic Network (poster) 


\section{PERSONNEL SUPPORTED (1989-2004)}

\section{Postdoctoral Associates}

Ying-Hong Li (1988 - 90), Financial Industry

\section{Graduate Students}

Jong-Rim Lee (Ph.D. 1993), Industry, Korea

Marcel Franz (Ph.D. 1994), Assist. Prof. of Physics, Univer. British Colombia

Calin Ciordas (M.S. 1996), Financial Industry

Tao Chen (Ph.D. 1997), Publishing Industry

Pramod Gupta (Ph.D. 1998), IBM, Toronto

Emily Clark (no degree), unknown

Violeta Gotcheva (Ph.D. 2004), Systems Analyst, Lab. for Laser Energetics, U. Rochester

Yanting Wang (Ph.D. 2004), Postdoctoral Assoc. in Chemistry, Univer. of Utah

\section{Undergraduate Students}

John Chiu (B.S. 1990), Graduate School in Physics, Caltech

Aihou Zhou (B.S. 1994), Graduate School in Elec. Engr., Cornell

Albert T. J. Wang (B.S. 2001), Graduate School in Physics, MIT

Thomas Goodman (B.S. 2004, Bucknell Univer.), Graduate School in Elec. Engr., Bucknell

\section{HONORS AND DISTINCTIONS}

John Chiu, 1990 Stoddard Prize for best senior thesis, Department of Physics and Astronomy, University of Rochester

Albert T. J. Wang, 2001 Stoddard Prize for best senior thesis, Department of Physics and Astronomy, University of Rochester

Albert T. J. Wang, 2nd Prize in Poster Competition, Meeting of the New York State Section of the American Physical Society, Buffalo, NY 10/21/00

Albert T. J. Wang, finalist for the 2002 Apker Prize of the American Physical Society "To recognize outstanding achievements in physics by undergraduate students, and thereby provide encouragement to young physicists who have demonstrated great potential for future scientific accomplishment"

S. Teitel, elected fellow of the American Physical Society in 2000, "For contributions towards the understanding and numerical modeling of critical phenomena in Josephson junction arrays and high-temperature superconductors". 